\title{
High-resolution lenses for sub-100 nm x-ray fluorescence microscopy
}

\author{
C. Davida) \\ Laboratory for Micro- and Nanotechnology, Paul Scherrer Institute, CH5232 Villigen-PSI, Switzerland \\ B. Kaulich, R. Barrett, M. Salomé, and J. Susini \\ European Synchrotron Radiation Facility, B. P. 220, F-38043 Grenoble Cedex, France
}

(Received 31 July 2000; accepted for publication 2 October 2000)

\begin{abstract}
We report on the design, fabrication, and testing of Fresnel zone plates for high-resolution x-ray fluorescence microscopy using the scanning x-ray microscope at the European Synchrotron Radiation Source. The germanium lenses were optimized for operation near the sulphur absorption edge at $2472 \mathrm{eV}$ photon energy. The high measured diffraction efficiencies of up to $9.6 \%$ and the good match to the spatial coherence of the undulator beam resulted in a photon flux of about 4 $\times 10^{8}$ photons per second within the bandwidth of a silicon $\langle 111\rangle$ monochromator. Using a test object consisting of zinc sulphide nanostructures, we were able to image features in sulphur x-ray fluorescence mode with lateral dimensions down to below $100 \mathrm{~nm}$. (C) 2000 American Institute of Physics. [S0003-6951(00)01149-9]
\end{abstract}

The availability of tunable undulator sources at high brilliance third generation synchrotrons such as the European Synchrotron Radiation Facility (ESRF) offers the possibility to produce submicron focused $\mathrm{x}$-ray beams with high flux and monochromaticity, in the few kilo-electron-volt energy range. For many samples the fluorescent x-ray emission resulting from absorption of the incident beam offers useful chemically sensitive information about the system under study. In particular, the vastly reduced Brems-strahlung spectral background confers a trace element sensitivity which can be significantly better than that possible using electron excitation. Furthermore, combining this detection scheme with absorption edge spectroscopy allows information to be obtained regarding the chemical environment and bonding state of particular elemental species present either in small quantities or in dilute concentrations.

Use of this fluorescence emission allows the study of bulk, nonconducting samples and is therefore also of interest for concentrated systems which cannot be prepared in suitably thin sections for standard absorption contrast microscopy. The characteristics of the ESRF ID21 x-ray microscopy beam line mean that it is well suited to applying $\mathrm{x}$-ray fluorescence microscopy for studies of sulphur content using both spectrometric and spectroscopic methods in applications such as geochemistry and human biology. ${ }^{1}$

The experimental methods being developed depend upon the quality of the microfocusing optics which must deliver high fluxes into the smallest possible probe size. The most effective focusing optics (in terms of efficiency and resolution) which have been developed for use at energies around $2.4 \mathrm{keV}$ are Fresnel zone plates. In this communication we describe the development of optimized high resolution zone plate optics and their subsequent characterization using sulphur containing test objects and $K$-line fluorescence contrast in a scanning $\mathrm{x}$-ray microscope (SXM). For high resolution and photon flux, a number of considerations for the design of optimized x-ray lenses have to be take into account.

${ }^{a)}$ Electronic mail: christian.david@psi.ch
The diffraction-limited resolving power of a zone plate depends on its outermost zone width, $d r_{n}$. The resolution $\delta_{R}$ according to the Rayleigh criterion is given by $\delta_{R}$ $=1.22 d r_{n}$. The Rayleigh model describes the imaging of two infinitely small incoherently illuminated point sources. The resolution limit is reached when the intensity dip $I_{\min }$ between the two superimposed Airy disks in the image amounts to $73.5 \%$ of the maximum intensity $I_{\max } \cdot{ }^{2}$ In this case, the contrast $\left(I_{\max }-I_{\min }\right) /\left(I_{\max }+I_{\min }\right)$ is $15.3 \%$. Although this Rayleigh contrast is somewhat arbitrary, as many microscopes can resolve smaller contrasts, it is useful to have a generally accepted criterion for the benchmarking of zone plates. The testing of zone plates in a SXM according to this model would require an object consisting of pairs of bright spots with diameters which are small compared to the expected resolution on a dark background. As such objects are difficult to obtain, resolution tests are often performed by imaging grating structures. The half pitch observable with the Rayleigh contrast of $15.3 \%$ is $\delta_{\text {grid }}=0.7 d r_{n} .{ }^{3}$ This means, that the diffraction limit of zone plates allows imaging of periodic structures with widths less than one outermost zone width.

The diffraction efficiency, i.e., the fraction of incident radiation that is diffracted into the order used for imaging, depends on the shape of the zone structures and the optical constants of the zone plate material. The material should have a strong phase shift, to avoid high aspect ratios of the outermost structures, coupled with low absorption. Excellent zone plates have been fabricated by electroplating of nickel into polymer molds. ${ }^{4-6}$ Semiconductors are also commonly used as they can be structured with high aspect ratios by plasma etching. ${ }^{7-10} \mathrm{We}$ chose germanium which, at $2.4 \mathrm{keV}$, allows up to $23 \%$ of the incoming radiation to be diffracted into the focal spot. However, this would require a structure height of $1350 \mathrm{~nm}$ (Fig. 1), leading to a very challenging aspect ratio of 13.5 for $100 \mathrm{~nm}$ wide outermost zones. Therefore, the zone height was chosen to be $900 \mathrm{~nm}$, limiting the obtainable efficiency to $18.3 \%$.

The flux that can be achieved in the focal spot of the lens 


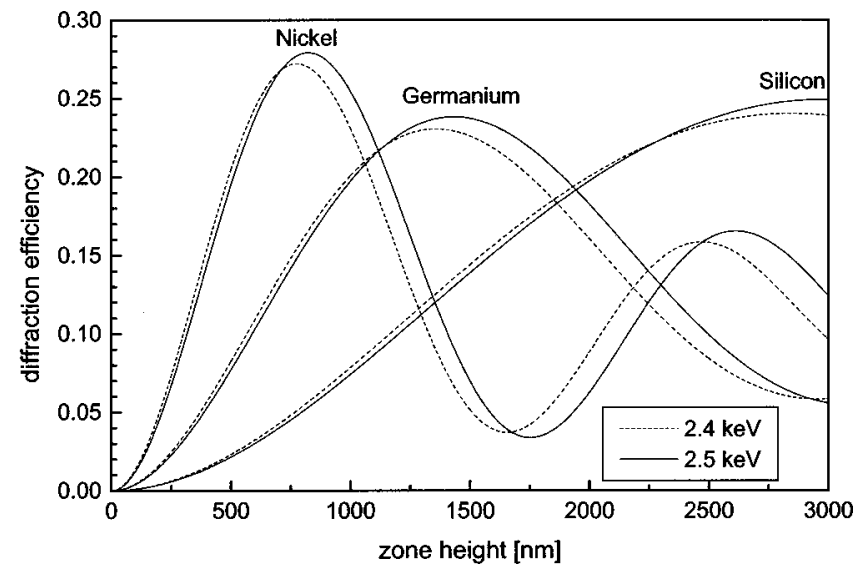

FIG. 1. First order diffraction efficiency of $\mathrm{Ni}, \mathrm{Si}$, and $\mathrm{Ge}$ zone plates with a square wave zone profile for photon energies near the $S K$ edge. The calculations are based on scalar diffraction theory (see Ref. 11).

also depends on the light collecting area. For diffraction limited probe sizes the maximum useful diameter of the zone plate, $d$, depends on the degree of spatial coherence provided by the source. To avoid a deterioration of the zone plate resolving power, the geometrical source image size $S^{\prime}$ should be well below the zone plate diffraction limit. For large demagnification factors, $S^{\prime}$ is given by $S^{\prime} \sim S f / g$, where $S$ is the effective source size, $f$ is the zone plates focal length, and $g$ is the effective source-to-zone plate distance. With $f \lambda=d r_{n} d$ and the size of the diffraction limited spot $\delta \sim d r_{n}$, this leads to a condition for diffraction limited imaging that is independent of the outermost zone width

$$
d<g \lambda / S .
$$

The maximum value of $d$ describes the size of the coherently illuminated area in the zone plate plane.

In the SXM at the beam line ID21 of ESRF, an undulator with a full width half maximum source size of $S_{h}=140 \mu \mathrm{m}$ (horizontal) $\times S_{v}=25 \mu \mathrm{m}$ (vertical) is demagnified by the zone plate situated $g=51 \mathrm{~m}$ from the source. For a photon energy of $2.4 \mathrm{keV}(\lambda \sim 5 \AA)$ the zone plate diameter should therefore be less than $1000 \mu \mathrm{m}$ to provide diffraction limited resolution in the vertical direction. In the horizontal direction, only $180 \mu \mathrm{m}$ of the zone plate is coherently illuminated. In order to have reasonable count rates, the zone plate diameters were chosen to be 300,400 , and $500 \mu \mathrm{m}$ (with respective focal lengths of 60,80 , and $100 \mathrm{~mm}$ at $2.4 \mathrm{keV}$ ). In consequence, diffraction limited resolution can only be expected in the vertical direction.

The main difficulties in the fabrication process of a zone plate are twofold. First, the pattern generation has to provide high resolution and high placement accuracy for a large number of curved lines with continuously varying linewidth. Second, the structures have to be transferred into the phase shifting material with high aspect ratios. The zone plates are supported by 250 -nm-thick silicon nitride membranes which have a transmission of $90 \%$ at $2.4 \mathrm{keV}$ photon energy. The membranes are coated by thermal evaporation with a $10-\mathrm{nm}$ thick adhesive layer of chromium, $900 \mathrm{~nm}$ of germanium as phase shifting material, and a 30-nm-thick top layer of chromium. A 60 -nm-thick layer of poly-methylmethacrylate is spincoated as an electron beam resist.

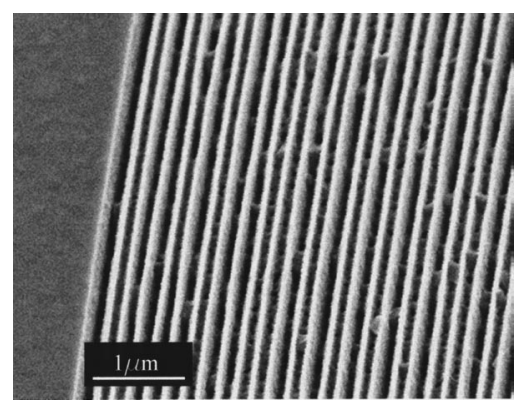

FIG. 2. Outermost zone structures etched into 900-nm-thick germanium. The outermost zone width is $100 \mathrm{~nm}$.

The electron beam exposures were done with a LION electron-beam lithography system from Leica Microsystems Jena. The LION has some special features which make it particularly suited for the generation of optical patterns. Its continuous path control mode allows the exposure of curved or straight lines by moving the sample stage continuously underneath the electron beam. This makes it possible to generate patterns over large areas without any field stitching. Each zone is exposed by a single sweep of the electron beam. The linewidth is individually controlled for each zone by adjusting the stage speed and the defocus of the beam. ${ }^{12}$ The resist pattern is first transferred into the 30-nm-thick Cr layer by reactive ion etching in a $\mathrm{Cl}_{2} / \mathrm{CO}_{2}$ plasma. The thin $\mathrm{Cr}$ layer then serves as a hard mask for a second etching step into the Ge layer using a $\mathrm{CBrF}_{3}$ plasma. Figure 2 shows scanning electron micrographs of the resulting Ge structures. The diffraction efficiency of the zone plates was measured to be up to $9.6 \%$ at $2.4 \mathrm{keV}$ photon energy. Taking into account the absorption by the support membrane, this corresponds to about $60 \%$ of the theoretical value, giving a peak flux of 4 $\times 10^{8} \mathrm{ph} / \mathrm{s}$ of radiation from a double crystal $\mathrm{Si}\langle 111\rangle$ monochromator $\left(5 \times 10^{-4}\right.$ relative bandwidth) at $180 \mathrm{~mA}$ ring current.

The resolution of a $300 \mu \mathrm{m}$ diameter Fresnel lens with $100 \mathrm{~nm}$ wide outermost zones was tested at $2.4 \mathrm{keV}$ photon energy in vacuum $\left(10^{-5} \mathrm{mbar}\right)$. We used a test object with approximately $350-\mathrm{nm}$-thick electroplated gold structures on a thin Si support membrane. The Au transmission is approximately $16 \%$ resulting in an object contrast of $64 \%$. Using a photodiode, the transmitted radiation was detected. The grating lines were positioned in horizontal direction to take advantage of the smaller vertical spot size. The narrowest lines with a half pitch of $85 \mathrm{~nm}$ can clearly be resolved (see Fig. 3).

To test the properties of the ID21 SXM using sulphur $K$ line $\mathrm{x}$-ray fluorescence emission as the imaging signal, zinc sulphide $(\mathrm{ZnS})$ siemens stars were generated by electron beam lithography and lift-off techniques. The structures shown here have a thickness of 40 or $26 \mathrm{~nm}$, their structure widths vary from $1 \mu \mathrm{m}$ down to $50 \mathrm{~nm}$ (see Fig. 4). The sample was scanned with radiation just above the sulphur $K$-absorption edge at $2472 \mathrm{eV}$. To limit the background signal from the uncoated regions, a Ge substrate was chosen.

The sulphur $K$-lines x-ray fluorescence $(2307-2464 \mathrm{eV})$ was collected using an energy dispersive $30 \mathrm{~mm}^{2}$ high-purity Ge detector (Princeton Gamma-Tech) equipped with a thin polymer entrance window. The detector was mounted in the 


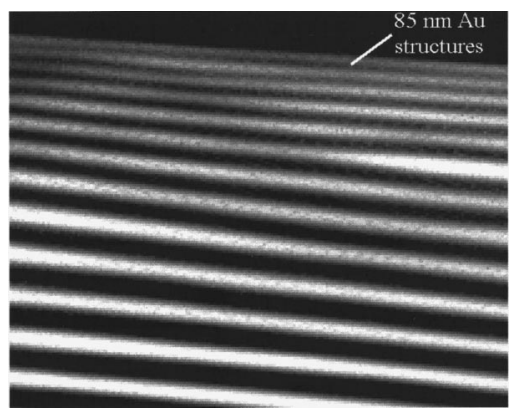

FIG. 3. Scanning x-ray microscope absorption contrast image of an approximately 350-nm-thick Au test object taken in transmission mode at $2.4 \mathrm{keV}$ photon energy. A $300 \mu \mathrm{m}$ diameter zone plate with $100 \mathrm{~nm}$ outermost structures was used. The test object was provided by M. Panitz, University of Göttingen

horizontal plane perpendicular to the incident beam to minimize the contribution of elastically scattered primary $\mathrm{x}$ rays. The sample was inclined towards the detector $\left(\sim 15^{\circ}\right)$ to allow the fluorescence signal to enter the detector. The signal was fed into a single channel analyser and pulses corresponding to the arrival of an $\mathrm{x}$ ray in the energy range appropriate for sulphur $K$ line emission were input to a fast scanning system.

Scanning x-ray fluorescence images of the $\mathrm{ZnS}$ test structures are shown in Fig. 5. The fluorescence yield for $S$ $K$-line emission is only $0.078 .{ }^{13}$ Given the collection solid angle ( $0.6 \%$ of $4 \pi$ steradians), the absorption of the $26-\mathrm{nm}$ thick $\mathrm{ZnS}$ film $(1.7 \%)$ and detector window transmission $(75 \%)$ we would therefore expect approximately $100 \mathrm{ph} / \mathrm{s}$ in the $S$ emission peaks when the probe is entirely incident on the $\mathrm{ZnS}$ structures. This value is in close agreement with the experimentally observed values. Considering a $100 \mathrm{~nm}$ diameter probe this signal originates from less than 3 $\times 10^{-16} \mathrm{~g}(300 \mathrm{ag})$ of sulphur and demonstrates the sensitivity of the method. Although the fluorescence signal is weak due to the reasons given earlier, the signal to background ratio from the outermost $1 \mu \mathrm{m}$ wide structures is about 10:1. This results in a contrast better than that of the Au test object imaged in transmission mode. The smallest structures that could be resolved have a half pitch of just below $100 \mathrm{~nm}$. However, close to the resolution limit the contrast is also deteriorated by significant shot noise.

Our studies have demonstrated how large aperture, high aspect ratio Ge zone plates can be used as probe forming
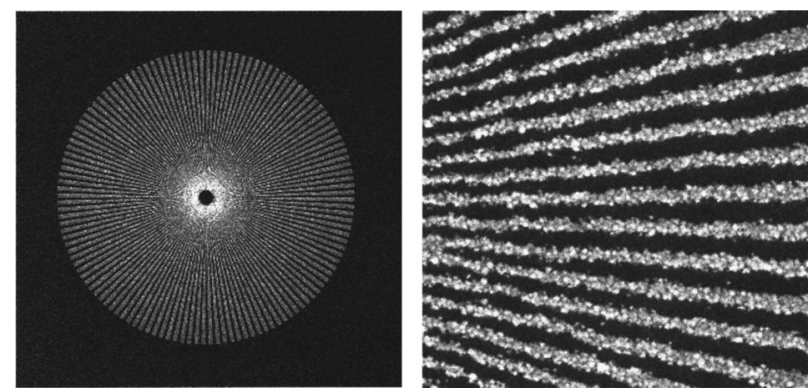

FIG. 4. SEM images of a ZnS Siemens star. The structures are $26 \mathrm{~nm}$ thick, the width ranges from $1 \mu \mathrm{m}$ down to $50 \mathrm{~nm}$ (half pitch). The right image shows structure widths from 175 to $75 \mathrm{~nm}$.
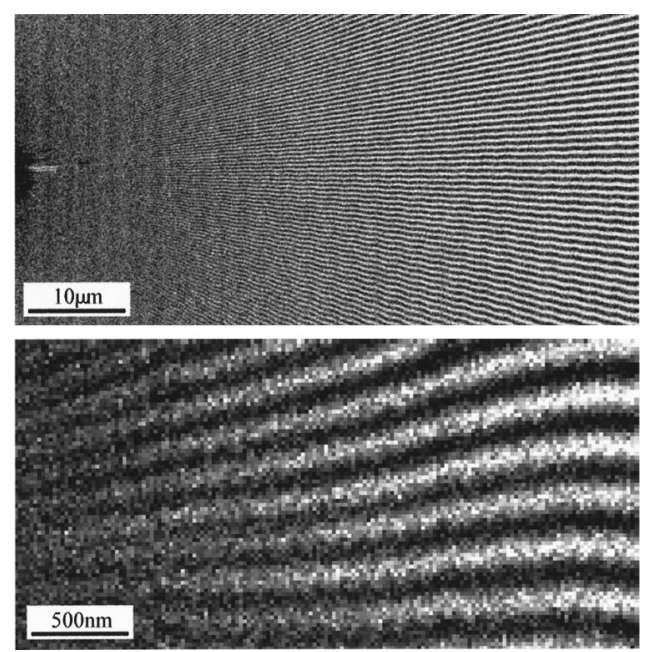

FIG. 5. SXM images of ZnS test structures imaged in fluorescence mode using a $300 \mu \mathrm{m}$ diameter zone plate with $100 \mathrm{~nm}$ outermost structures. Top image: 40-nm-thick structures imaged with $125 \mathrm{~nm}$ pixel distance and a dwell time of $100 \mathrm{~ms}$. Lower image: 26-nm-thick structures with half pitch values ranging from 175 down to $75 \mathrm{~nm}$. Pixel distance: $25 \mathrm{~nm}$, dwell time: $2 \mathrm{~s}$. Some image distortion is caused by beam movement during the scan.

optics for a SXM around $2.4 \mathrm{keV}$. Moreover, the high efficiencies which have been achieved give flux densities sufficiently high to allow the detection of characteristic fluorescence with sub-100 nm spatial resolution on model thin-layer systems even for a light element with low fluorescence yield. Future development of the technique will concentrate upon the application of $\mathrm{x}$-ray fluorescence microscopy to perform quantitative quantitative extended $\mathrm{x}$-ray absorption fine structure analysis of "real-life" systems that are more demanding in terms of spectral normalization.

The authors wish to thank M. Panitz for providing the gold test object shown in Fig. 3. G. Schmahl and M. Peuker are gratefully acknowledged for providing access to the reactive ion etcher used for the structuring of the germanium zone plates. The authors also thank the technical staff of LMN and ESRF for the excellent working conditions.

${ }^{1}$ R. Barrett, B. Kaulich, M. Salomé, and J. Susini, in X-ray Microscopy: 6th International Conference, edited by W. Meyer-Ilse, T. Warwick, and D. Attwood (AIP, Melville, NY, 2000), p. 458.

${ }^{2}$ M. Born and E. Wolf, Principles of Optics, 4th ed. (Pergamon, New York, 1970), p. 415.

${ }^{3}$ D. Attwood, Soft X-Rays and Extreme Ultraviolet Radiation (Cambridge University Press, Cambridge, 1999), p. 357.

${ }^{4}$ E. Anderson and D. Kern, in X-Ray Microscopy III, edited by A. G. Michette, G. R. Morrison, and C. J. Buckley (Springer, Berlin, 1990), p. 75.

${ }^{5}$ D. Weiss, M. Peuker, and G. Schneider, Appl. Phys. Lett. 72, 1805 (1998).

${ }^{6}$ E. Di Fabrizio, F. Romanato, M. Gentili, S. Cabrini, B. Kaulich, J. Susini, and R. Barrett, Nature (London) 401, 895 (1999).

${ }^{7}$ C. David and A. Souvorov, Rev. Sci. Instrum. 70, 4168 (1999).

${ }^{8}$ G. Schneider, T. Schliebe, and H. Aschoff, J. Vac. Sci. Technol. B 13, 2809 (1995).

${ }^{9}$ S. J. Spector, C. J. Jacobsen, and D. M. Tennant, J. Vac. Sci. Technol. B 15, 2872 (1997).

${ }^{10}$ C. David, J. Thieme, P. Guttmann, G. Schneider, D. Rudolph, and G. Schmahl, Optik (Stuttgart) 91, 95 (1992).

${ }^{11}$ J. Kirz, J. Opt. Soc. Am. 1, 301 (1974).

${ }^{12}$ C. David and D. Hambach, Microelectron. Eng. 46, 219 (1999).

${ }^{13}$ M. O. Krause, J. Phys. Chem. Ref. Data 8, 307 (1979). 